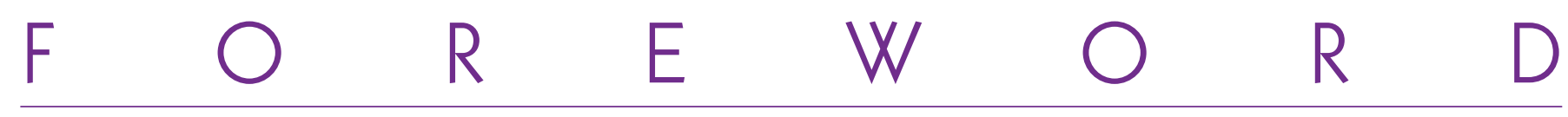

\title{
Qualified Dentofacial Orthodontic Specialist
}

\author{
F. Flageul
}

"In this day and age, what are perfect delays?"

Paul Valéry

Mauvaises pensées

This issue is themed "eclecticism" because it is aimed at discussing wide-ranging topics os that the diverse wishes of the readers are satisfied.

Clinicians can ensure effective and reproducible clinical practice by constantly keeping themselves up-to-date on fundamental and clinical research. It requires interdisciplinary collaboration, which is now integrated into treatment processes. The authors attest to this in these pages.

Marc Bert shares knowledge on the peri-implant gingiva through the study of its anatomy, physiology, and development. After a concise and precise review of the biology of the periodontium and histology of the gingival-implant sulcus, he demonstrates its resemblance to the epithelial-mucosal attachment of the tooth. He describes the maintenance of the quality of the tissues surrounding the implant and even its improvement over time, with the possibility of strengthening the keratinized gingiva or its creation, provided oral hygiene is satisfactory.

This is not the case for implants that are used to stabilize removable prostheses.

Fabienne and Frank Pourrat state that the diode laser is insufficiently used by orthodontists. They propose its use in (a) beautifying the smile with a microsurgery for soft-tissue enhancement and (b) increasing the stability of malposition corrections by decreasing malposition recurrence.

It is through a comprehensive decision tree that a practitioner can assess the potential of gingivectomies to contribute to soft-tissue esthetics.

Address for correspondence:

Françoise Flageul - 29b rue des Francs Bourgeois -

75004 Paris

E-mail: soizic.flageul@wanadoo.fr

Article received: 26-11-2017. Accepted for publication: 15-02-2018.

This is an Open Access article distributed under the terms of the Creative Commons Attribution License (http://creativecommons.org/licenses/by/4.0), which permits unrestricted use, distribution, and reproduction in any medium, provided the original work is properly cited. 
For preventing malposition recurrence, they recommend performing a rotation by a circumferential fibrotomy of the tooth and the reopening of extraction spaces by the removal of the interdental gingiva, always using the laser.

Hélène Gil reminds us how important the contribution of the physoitherapist is in the diagnosis and treatment of pain and dysfunctional disorders of the mandibular system.

She focuses on challenging the pure occlusive etiology of TMDs and stresses the importance of muscular disharmony and stress in the onset of pain, with psychosocial factors also being taken into account.

Global behavioral treatment combines linguistic training according to the Fournier method with Schultz autogenous training.

Only the combination of training and relaxation gives the patient means to manage their muscular tension.

Jean-Louis Raymond proposes a systemic approach to the treatment of severe class-II malocclusions by overcoming the difficulty of their correction by the use of fixed anterior elevations. With the patient's cooperation, it is possible to correct the anatomical anomaly (overhang and supraclusion) while introducing new chewing cycles that will help perpetuate the result obtained. The resulting morphological correction is stable in the long term because the three-dimensional shape of arches. In particular, the spatial orientation arches is compatible with effective physiological mastication, which can be taught using a natural restraint device.

A clinical case of managing the horizontal inclusion of two maxillary incisors is proposed by Hamza Ben Aissa and colleagues.

The authors stress the need to address these inclusions as early as possible because of the esthetic complications they cause and the role of these anterior teeth in guiding the occlusion.

They highlight the difficulties of diagnosis, facilitated by the use of conebeam computed tomography, and treatment that combines the removal of possible obstacles, the creation of the necessary space, and rigorous therapeutic monitoring.

Chloé Bernard Granger presents another clinical proposals. She favors single-phase surgery to treat the maxillary insufficiency of an adult patient with a highly divergent class-III malocclusion pattern. She reports that a multilingual attachment combined with a $\mathrm{FKS}^{\circledR}$ disjunctor allows for reliable and technically simple expansion.

Classically, the disjunction is carried out first during the operation. However, it is possible to manage the perform a procedure in all thee spatial dimensions. The lingual disjunctor presented in this study is a significant step forward in the treatment and management of lingual orthodontic surgery cases.

The determinating factor of its stability appears to be the occlusion obtained immediately after surgery.

We would like to thank Hélène Guiral-Desnoës, who diligently managed the press review, supplementing her analyses with personal remarks that encouraged a fruitful discussion.

Happy reading! 